\title{
RESEARCH
}

Open Access

\section{Myocardial tissue and metabolism characterization in men with alcohol consumption by cardiovascular magnetic resonance and 11C-acetate PET/CT}

Shuai Liu', Xue Lin ${ }^{2}$, Ximin Shi ${ }^{3,4}$, Ligang Fang ${ }^{2}$, Li Huo ${ }^{3,4^{*+}}$, Fei Shang ${ }^{5}$, Juhani Knuuti ${ }^{6}$, Chunlei Han ${ }^{6}$, Xiaomeng Wu ${ }^{5}$, Rui Guo ${ }^{1}$, Haiyan Ding ${ }^{1}$, Runhua Zhang ${ }^{7}$, Huimin Duan ${ }^{8}$, Jie Ding ${ }^{3,4}$, Haiqun Xing ${ }^{3,4}$ and Xihai Zhao ${ }^{1 *+}$

\begin{abstract}
Background: Chronic alcohol consumption initially leads to asymptomatic left ventricular dysfunction, but can result in myocardial impairment and heart failure if ongoing. This study sought to characterize myocardial tissues and oxidative metabolism in asymptomatic subjects with chronic alcohol consumption by quantitative cardiovascular magnetic resonance (CMR) and 11C-acetate positron emission tomography (PET)/computed tomography (CT).

Methods: Thirty-four male subjects ( $48.8 \pm 9.1$ years) with alcohol consumption $>28 \mathrm{~g} /$ day for $>10$ years and 35 age-matched healthy male subjects (49.5 \pm 9.7 years) underwent CMR and 11C-acetate PET/CT. Native and post T1 values and extracellular volume (ECV) from CMR and Kmono and K1 from PET imaging were measured. Quantitative measurements by CMR and PET imaging were compared between subjects with moderate to heavy alcohol consumption and healthy controls, and their correlations were also analyzed.

(Continued on next page)
\end{abstract}

\footnotetext{
* Correspondence: HuoLi@pumch.cn; xihaizhao@tsinghua.edu.cn

+Xihai Zhao and Li Huo are co-corresponding authors and equally contributed to this study.

${ }^{3}$ Department of Nuclear Medicine, Peking Union Medical College Hospital, Chinese Academy of Medical Science, 1\# Shuaifuyuan, Dongcheng District, Beijing 100730, China

${ }^{1}$ Center for Biomedical Imaging Research, Department of Biomedical

Engineering, Tsinghua University School of Medicine, Haidian District, Beijing 100084, China

Full list of author information is available at the end of the article
}

(c) The Author(s). 2020 Open Access This article is licensed under a Creative Commons Attribution 4.0 International License, which permits use, sharing, adaptation, distribution and reproduction in any medium or format, as long as you give appropriate credit to the original author(s) and the source, provide a link to the Creative Commons licence, and indicate if changes were made. The images or other third party material in this article are included in the article's Creative Commons licence, unless indicated otherwise in a credit line to the material. If material is not included in the article's Creative Commons licence and your intended use is not permitted by statutory regulation or exceeds the permitted use, you will need to obtain permission directly from the copyright holder. To view a copy of this licence, visit http://creativecommons.org/licenses/by/4.0/ The Creative Commons Public Domain Dedication waiver (http://creativecommons.org/publicdomain/zero/1.0/) applies to the data made available in this article, unless otherwise stated in a credit line to the data. 
(Continued from previous page)

Results: Compared to healthy controls, subjects with alcohol consumption showed significantly shorter native T1 $(1133 \pm 65$ ms vs. $1186 \pm 31$ ms, $p<0.001)$ and post T1 (477 \pm 42 ms vs. $501 \pm 38$ ms, $p=0.008)$ values, greater ECV $(28.2 \pm 2.2 \%$ vs. $26.9 \pm 1.3 \%, p=0.003)$, marginally lower Kmono $\left(57.6 \pm 12.1 \mathrm{~min}^{-1} \times 10^{-3}\right.$ vs. $63.0 \pm 11.7 \mathrm{~min}^{-1} \times$ $\left.10^{-3}, p=0.055\right)$, and similar K1 (0.82 $\pm 0.13 \mathrm{~min}^{-1}$ vs. $\left.0.83 \pm 0.15 \mathrm{~min}^{-1}, p=0.548\right)$ after adjusting for confounding factors. There were no significant differences in CMR measurements and K1 between subjects with heavy and moderate alcohol consumption (all $p>0.05$ ). In contrast, subjects with heavy alcohol consumption showed significantly lower Kmono values compared to those with moderate alcohol consumption $\left(52.9 \pm 12.1 \mathrm{~min}^{-1} \times 10^{-3}\right.$ vs. $\left.63.7 \pm 9.2 \mathrm{~min}^{-1} \times 10^{-3}, p=0.012\right)$. Strong and moderate correlations were found between $\mathrm{K} 1$ and ECV in healthy controls $(r=0.689, p=0.013)$ and subjects with moderate alcohol consumption $(r=0.518, p=0.048)$, respectively.

Conclusion: Asymptomatic men with heavy alcohol consumption have detectable structural and metabolic changes in myocardium on CMR and 11C-acetate PET/CT. Compared with quantitative CMR, 11C-acetate PET/CT imaging may be more sensitive for detecting differences in myocardial damage among subjects with moderate to heavy alcohol consumption.

Keywords: Alcoholic cardiomyopathy, T1 mapping, Magnetic resonance imaging, Oxidative metabolism, Positron emission tomography

\section{Introduction}

Alcohol consumption is the third leading lifestyle-related cause of death for people in the US, behind tobacco and improper diet/lack of physical activity, and is responsible for $3.8 \%$ of all deaths globally [1-3]. Alcoholic cardiomyopathy accounts for up to $40 \%$ of patients with non-ischemic dilated cardiomyopathy [4]. Without complete abstinence, the 4-year mortality rate for alcohol-induced dilated cardiomyopathy approaches 50\% [5]. Although moderate alcohol consumption may not be harmful to the cardiovascular system, excessive intake of alcohol can damage the myocardium via stimulating the apoptosis of cardiomyocytes and inhibiting energy metabolism [6-9]. Initially, alcohol consumption leads to asymptomatic left ventricular (LV) dysfunction, and can result in symptoms of heart failure if continues. Therefore, early assessment of the changes in structure and metabolism of myocardial tissues in subjects with alcoholism at asymptomatic stage is important for prevention of irreversible outcomes, such as dilated cardiomyopathy and heart failure.

Quantitative cardiovascular magnetic resonance (CMR) imaging, such as T1 mapping, can measure the native value of longitudinal (spin-lattice) relaxation time (T1) of myocardial tissues and extracellular volume (ECV), which represents the size of the extracellular space and reflects interstitial diseases [10]. The native T1 values of myocardial tissues vary with myocardial extracellular water (edema), focal or diffuse fibrosis, fat, iron, and amyloid proteins. Meanwhile, quantitative parameters measured from 11C-acetate positron emission tomography (PET) imaging, such as Kmono and $\mathrm{K} 1$, have been largely utilized to characterize myocardial metabolism in heart diseases [11-14]. Kmono is considered as a robust marker for evaluation of myocardial oxygen consumption
$\left(\mathrm{MVO}_{2}\right)$, while $\mathrm{K} 1$ is correlated with myocardial blood flow (MBF) in absolute terms. As such, it is possible to assess changes in structure and metabolism of myocardial tissues in subjects with alcohol consumption by combining quantitative $\mathrm{CMR}$ with $11 \mathrm{C}$-acetate $\mathrm{PET} /$ computed tomography (CT) imaging.

This study sought to investigate tissue characterization and metabolic disorders in asymptomatic subjects with chronic alcohol consumption by using quantitative CMR and $11 \mathrm{C}$-acetate PET/CT imaging.

\section{Methods}

Study sample

Male adults with a history of drinking $>10$ years and average alcohol consumption $>2$ std/day $(1$ std $/$ day $=14 \mathrm{~g}$ pure ethanol) were recruited. Subjects with one of the following conditions were excluded: 1) hypertension; 2) diabetes mellitus; 3) coronary artery disease; 4) LV ejection fraction (LVEF) $<50 \%$ or abnormalities in cardiac structure on ultrasound imaging; 5) congenital heart disease; 6) renal dysfunction (glomerular filtration rate $<60 \mathrm{~mL} / \mathrm{min}$ ); 7) atrial fibrillation or premature beat on electrocardiogram; 8) cirrhosis; and 9) contraindications to CMR examination. Healthy male adults with matched ages but without history of alcohol consumption or with only occasional alcohol intake $(<100 \mathrm{~g} /$ month) were enrolled as healthy controls. The following demographic and clinical information was collected: age, height, weight, blood pressure, heartbeat, and history of smoking (current or one-time smoker). A questionnaire was conducted to collect the following information on alcohol consumption: 1) how many times do you drink in a week? 2) which type of alcoholic beverage do you drink, e.g. beer, wine or liquor? 3) how much of alcoholic beverage do you drink per drinks? 4) how many 
years have you maintained this type of drinking? The study protocol was approved by Institutional Review Board of Tsinghua University School of Medicine and a signed consent form was obtained from each subject prior to enrollment.

\section{CMR imaging}

CMR imaging was performed on a 3 Tesla scanner (Achieva TX, Philips Healthcare, Best, The Netherlands) with a 32-channel cardiac coil. The CMR protocol included CINE, pre- and post-contrast enhanced T1 mapping. The image parameters were as follows: CINE: turbo field echo; repetition time/echo time $3.2 / 1.6 \mathrm{~ms}$, field of view $320 \times 320 \mathrm{~mm}^{2}$, flip angle $40^{\circ}$, and slice thickness $8 \mathrm{~mm}$; and T1-mapping: 3-3-5 modified LookLocker inversion recovery (MOLLI), repetition time/ echo time $2.3 / 0.9 \mathrm{~ms}$, field of view $320 \times 320 \mathrm{~mm}^{2}$, flip angle $35^{\circ}$, and slice thickness $8 \mathrm{~mm}$. For CINE imaging, multiple slices were acquired on short-axis view. T1 mapping was acquired on three short-axis views of the mid ventricular septum and $10 \mathrm{~mm}$ up and down with breath holding. Post contrast enhanced T1-mapping was conducted 10 min after administration of a Gadoliniumbased contrast agent (Magnevist, Bayer Schering Pharma, Berlin, Germany) with a dose of $0.15 \mathrm{mmol} / \mathrm{kg}$ and a flow rate of $2 \mathrm{ml} / \mathrm{s}$.

\section{PET/CT imaging}

PET/CT imaging was performed on a hybrid PET/CT scanner (PoleStar m660, Sinounion, Healthcare Inc., Beijing, China). After a low dose CT scanning for location $(120 \mathrm{kV}$ and $140 \mathrm{~mA}), 11 \mathrm{C}$-acetate with a dose of 740 $\mathrm{MBq}$ was injected intravenously, followed by a $40-\mathrm{min}$ dynamic PET scan. A total of 53 frames $(15 \times 10 \mathrm{~s}, 15 \times$ $30 \mathrm{~s}, 16 \times 60 \mathrm{~s}$, and $7 \times 120 \mathrm{~s}$ ) were reconstructed using the 3D OSEM+TOF algorithm on a Precision workstation (Sinounion, Healthcare Inc., Beijing, China) with an object space of $192 \times 192 \times 117$ and a voxel size of $3.15 \times 3.15 \times$ $1.87 \mathrm{~mm}^{3}$ after correction for dead time, decay and measured photon attenuation.

\section{Data analysis}

CMR images were analyzed with group-wise registration using the open source toolbox Elastix and non-linear curve fitting based on the Levenberg-Marquardt algorithm was then performed. Two radiologists who had $>5$ years' experience in CMR measured the values for LV and right ventricular (RV) end diastolic volumes (LVEDV and RVEDV), left and right ventricular end systolic volumes (LVESV and RVESV), LV and RV stroke volumes (LVSV and RVSV), LVEF, RV ejection fraction (RVEF), cardiac output (CO), and LV mass using a CMR workstation (Philips Extended MR WorkSpace 2.6.3.4). The values of native $\mathrm{T} 1$, post $\mathrm{T} 1$ and $\mathrm{ECV}$ at the middle interventricular septum on three slices were measured by two radiologists. The mean values of native T1, post T1 and ECV on three slices were then recorded. The radiologists were blinded to history of alcohol consumption and PET/CT imaging data.

PET/CT images were reviewed by two reviewers with > 10 years` experience in nuclear medicine who were blinded to history of alcohol consumption and CMR imaging data. Kmono was calculated by monoexponential fitting and $\mathrm{K} 1$ was calculated using a singlecompartmental model with LV time-activity curve as the input function. Both Kmono and K1 were calculated at two levels: global for the entire myocardium and segmented for the 17-segment model [15]. All analyses were performed using the Carimas software (v2.9, developed in Turku PET Centre of Finland, http://www.turkupetcentre. fi/carimas). A total of 20 subjects with alcohol consumption were randomly selected for testing the reproducibility of Kmono and K1 measurements. Each observer reviewed PET/CT images using Carimas independently, then performed reorientation, short axis definition and manual correction. One reviewer analyzed the data of PET/CT again after 1 month to minimize memory bias.

\section{Statistical analysis}

Continuous variables were presented as mean \pm standard deviation (SD). Kmono and K1 measurements in the septal segments (Seg8 and Seg9), corresponding to the same regions analyzed in CMR, were taken. The mean values for native T1, post T1, ECV, Kmono and $\mathrm{K} 1$ from the two reviewers were compared between alcohol consumption groups and healthy controls using an independent $t$ test. The linear relationship between measurements from CMR and PET imaging was determined using Spearman's correlation coefficient. For testing reproducibility, intraclass correlation coefficient (ICC) and a corresponding 95\% confidence interval (CI) were calculated to determine inter- and intra-observer agreement in measuring Kmono and K1. A $p<0.05$ was considered statistically significant and all statistical analysis was conducted with SPSS (v 25.0 Statistical Package for the Social Sciences, International Business Machines, Inc., Armonk, New York, USA).

\section{Results}

In total, 40 subjects with moderate to heavy alcohol consumption and 38 healthy controls were recruited for this study between February and December 2017. Six subjects with moderate to heavy alcohol consumption and 3 healthy controls were excluded due to the following reasons: 1) failure of contrast agent injection for CMR imaging $(n=1)$ or tracer for PET/CT scanning $(n=2)$; 2$)$ poor image quality for CMR imaging $(n=5)$; and 3) claustrophobia $(\mathrm{n}=1)$. Of the remaining 35 
healthy controls, only the first 12 (34.3\%) underwent PET imaging. Subjects with a history of alcohol consumption were divided into moderate $(<5 \mathrm{std} /$ day, $n=15$ ) and heavy consumption groups ( 25 std/day, $n=$ 19), according to the 2015 to 2020 Dietary Guidelines for Americans 8th Edition (mild consumption: <2std/ day; moderate consumption: 2-5std/day; heavy consumption: $\geq 5 \mathrm{std} /$ day. $1 \mathrm{std} /$ day $=14 \mathrm{~g}$ pure ethanol). Clinical characteristics and measurements from echocardiography are shown in Table 1. All subjects with moderate to heavy alcohol consumption and healthy controls were male with mean ages of $48.8 \pm 9.1$ and $49.5 \pm 9.7$ years $(p=0.771)$, respectively. Compared to healthy controls, subjects with moderate to heavy alcohol consumption exhibited significantly higher diastolic blood pressure $(77.6 \pm 8.3 \mathrm{mmHg}$ vs. $73.2 \pm 6.7 \mathrm{mmHg}$, $p=0.020)$, prevalence of smoking $(62.0 \%$ vs. $20.0 \%, p<$ $0.001)$, body mass index (BMI) $\left(26.0 \pm 2.9 \mathrm{~kg} / \mathrm{m}^{2}\right.$ vs. $\left.23.2 \pm 2.2 \mathrm{~kg} / \mathrm{m}^{2}, p<0.001\right)$, LVEDV $(116.2 \pm 21.5 \mathrm{ml}$ vs. $106.0 \pm 20.2 \mathrm{ml}, p=0.046)$, LVESV $(41.7 \pm 12.2 \mathrm{ml}$ vs. $35.3 \pm 7.6 \mathrm{ml}, p=0.010), \quad \operatorname{RVEDV}(130.3 \pm 24.5 \mathrm{ml}$ vs.
$117.5 \pm 26.3 \mathrm{ml}, p=0.041)$, and RVESV $(64.3 \pm 15.0 \mathrm{ml}$ vs. $54.6 \pm 13.2 \mathrm{ml}, p=0.006)$. No significant differences were found in systolic blood pressure, LVEF, RVEF, LVSV, RVSV, CO and LV mass (all $p>0.05$ ) between subjects with moderate to heavy alcohol consumption and healthy controls. No significant differences were observed in clinical characteristics between subjects with heavy and moderate alcohol consumption (all p >0.05).

\section{Comparison of CMR measurements}

Table 2 summarizes the comparison results of the quantitative measurements from CMR and PET imaging among subjects with heavy to moderate alcohol consumption, and those without alcohol consumption. Compared to healthy controls, subjects with moderate to heavy alcohol consumption showed significantly shorter native and post $\mathrm{T} 1$ values, and greater ECV (all $p<0.05$, Fig. 1). Significant differences were found in native T1 values $(p=0.004)$ between subjects with moderate alcohol consumption and healthy controls, but the differences in post $\mathrm{T} 1$

Table 1 Clinical characteristics of the study population

\begin{tabular}{|c|c|c|c|c|c|c|}
\hline & \multicolumn{4}{|c|}{ Mean \pm SD or $n(\%)$} & \multirow[t]{3}{*}{$\mathrm{P}^{\mathrm{a}}$} & \multirow[t]{3}{*}{$P^{b}$} \\
\hline & \multicolumn{3}{|c|}{ Subjects with moderate to heavy alcohol consumption } & \multirow{2}{*}{$\begin{array}{l}\text { Healthy } \\
\text { controls } \\
(n=35)\end{array}$} & & \\
\hline & $\begin{array}{l}\text { All } \\
(n=34)\end{array}$ & $\begin{array}{l}\text { Heavy consumption } \\
(n=19)\end{array}$ & $\begin{array}{l}\text { Moderate consumption } \\
(n=15)\end{array}$ & & & \\
\hline Age, years & $48.8 \pm 9.1$ & $49.9 \pm 11.1$ & $47.4 \pm 5.9$ & $49.5 \pm 9.7$ & 0.771 & 0.407 \\
\hline $\mathrm{BMl}, \mathrm{kg} / \mathrm{m}^{2}$ & $26.0 \pm 2.9$ & $25.8 \pm 3.1$ & $26.3 \pm 2.8$ & $23.2 \pm 2.2$ & $<0.001$ & 0.672 \\
\hline Smoke & $21(62.0)$ & $12(63.2)$ & $9(60.0)$ & $7(20.0)$ & $<0.001$ & 0.856 \\
\hline $\mathrm{SBP}, \mathrm{mmHg}$ & $120.0 \pm 11.2$ & $116.8 \pm 11.4$ & $124.1 \pm 9.8$ & $116.5 \pm 10.8$ & 0.192 & 0.055 \\
\hline $\mathrm{DBP}, \mathrm{mmHg}$ & $77.6 \pm 8.3$ & $76.5 \pm 8.3$ & $78.9 \pm 8.5$ & $73.2 \pm 6.7$ & 0.020 & 0.424 \\
\hline \multicolumn{7}{|c|}{ Alcohol consumption } \\
\hline Duration, years & $26.2 \pm 8.9$ & $26.5 \pm 10.7$ & $25.8 \pm 6.3$ & 0 & - & 0.806 \\
\hline Dose, g/day & $115.8 \pm 119.7$ & $167.2 \pm 140.6$ & $50.5 \pm 13.2$ & 0 & - & 0.002 \\
\hline \multicolumn{7}{|l|}{ Left ventricle } \\
\hline LVEDV, mL & $116.2 \pm 21.5$ & $115.4 \pm 25.4$ & $117.2 \pm 16.0$ & $106.0 \pm 20.2$ & 0.046 & 0.808 \\
\hline LVESV, mL & $41.7 \pm 12.2$ & $43.2 \pm 13.5$ & $39.8 \pm 10.5$ & $35.3 \pm 7.6$ & 0.010 & 0.425 \\
\hline LVSV, mL & $74.4 \pm 17.7$ & $72.1 \pm 18.5$ & $77.4 \pm 16.7$ & $70.7 \pm 16.7$ & 0.369 & 0.396 \\
\hline LVEF, \% & $64.9 \pm 6.7$ & $63.8 \pm 6.1$ & $66.3 \pm 7.3$ & $66.8 \pm 5.6$ & 0.219 & 0.282 \\
\hline $\mathrm{CO}, \mathrm{L} / \mathrm{min}$ & $5.0 \pm 1.0$ & $4.9 \pm 1.2$ & $5.2 \pm 0.8$ & $4.6 \pm 1.0$ & 0.073 & 0.312 \\
\hline LV mass, g & $112.1 \pm 19.5$ & $110.7 \pm 22.7$ & $113.9 \pm 15.2$ & $105.7 \pm 17.5$ & 0.158 & 0.646 \\
\hline \multicolumn{7}{|l|}{ Right ventricle } \\
\hline RVEDV, mL & $130.3 \pm 24.5$ & $130.5 \pm 25.7$ & $130.0 \pm 23.7$ & $117.5 \pm 26.3$ & 0.041 & 0.954 \\
\hline RVESV, mL & $64.3 \pm 15.0$ & $62.8 \pm 12.9$ & $65.5 \pm 16.6$ & $54.6 \pm 13.2$ & 0.006 & 0.610 \\
\hline RVSV, mL & $66.0 \pm 14.9$ & $67.3 \pm 18.1$ & $65.0 \pm 12.3$ & $63.0 \pm 18.7$ & 0.458 & 0.686 \\
\hline RVEF, \% & $50.7 \pm 6.8$ & $51.3 \pm 8.3$ & $50.2 \pm 5.4$ & $52.9 \pm 8.9$ & 0.251 & 0.654 \\
\hline
\end{tabular}

$B M I$ body mass index; SBP systolic blood pressure; DBP diastolic blood pressure; LVEDV left ventricular end diastolic volume; LVESV left ventricular end systolic volume; LVSV left ventricular stroke volume; $L V E F$ left ventricular ejection fraction; CO cardiac output; $L V$ mass left ventricular mass; RVEDV right ventricular end

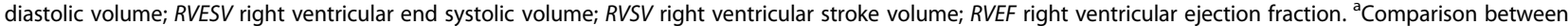
subjects with moderate to heavy alcohol consumption and healthy controls. ${ }^{b}$ Comparison between subjects with heavy and moderate alcohol consumption 
Table 2 Comparison between subjects with and without alcohol consumption

\begin{tabular}{|c|c|c|c|c|c|c|c|c|}
\hline & \multicolumn{4}{|c|}{ Mean \pm SD or $n(\%)$} & \multirow[t]{3}{*}{$p^{a}$} & \multirow[t]{3}{*}{$\mathrm{P}^{\mathrm{b}}$} & \multirow[t]{3}{*}{$p^{c}$} & \multirow[t]{3}{*}{$P^{d}$} \\
\hline & \multicolumn{3}{|c|}{ Subjects with alcohol consumption } & \multirow{2}{*}{$\begin{array}{l}\text { Healthy } \\
\text { controls } \\
(n=35)\end{array}$} & & & & \\
\hline & $\begin{array}{l}\text { All } \\
(n=34)\end{array}$ & $\begin{array}{l}\text { Heavy consumption } \\
(n=19)\end{array}$ & $\begin{array}{l}\text { Moderate consumption } \\
(n=15)\end{array}$ & & & & & \\
\hline \multicolumn{9}{|l|}{ CMR measurements } \\
\hline Native T1, ms & $1133 \pm 65$ & $1130 \pm 73$ & $1136 \pm 55$ & $1186 \pm 31$ & $<0.001$ & 0.004 & 0.004 & 0.791 \\
\hline Post T1, ms & $477 \pm 42$ & $464 \pm 45$ & $494 \pm 32$ & $501 \pm 38$ & 0.013 & 0.002 & 0.491 & 0.036 \\
\hline$E C V, \%$ & $28.2 \pm 2.2$ & $28.9 \pm 2.3$ & $27.3 \pm 1.9$ & $26.9 \pm 1.3$ & 0.004 & 0.001 & 0.377 & 0.034 \\
\hline \multicolumn{9}{|l|}{ PET measurements } \\
\hline Kmono, $\min ^{-1} \times 10^{-3}$ & $57.6 \pm 12.1$ & $52.9 \pm 12.1$ & $63.7 \pm 9.2$ & $63.0 \pm 11.7 \|$ & 0.189 & 0.028 & 0.861 & 0.007 \\
\hline $\mathrm{K} 1, \min ^{-1}$ & $0.82 \pm 0.13$ & $0.80 \pm 0.11$ & $0.84 \pm 0.16$ & $0.83 \pm 0.15 \|$ & 0.833 & 0.589 & 0.865 & 0.4 \\
\hline
\end{tabular}

ECV extracellular volume fraction. ${ }^{\mathrm{a} C}$ Comparison between subjects with moderate to heavy alcohol consumption and healthy controls. ${ }^{\mathrm{b}} \mathrm{Comparison}$ between subjects with heavy alcohol consumption and healthy controls. ${ }^{\circ}$ Comparison between subjects with moderate alcohol consumption and healthy controls.

${ }^{\mathrm{d}}$ Comparison between subjects with heavy and moderate alcohol consumption. ||The measurements were taken from 12 healthy controls

values and ECV were not statistically significant between these two groups (all $p>0.05$ ). In contrast, subjects with heavy alcohol consumption showed significantly shorter native and post $\mathrm{T} 1$ values, and greater ECV than healthy controls (all $p<0.05$ ). In addition, compared with subjects with moderate alcohol consumption, those with heavy alcohol consumption showed significantly shorter post T1 values $(p=$ $0.036)$ and greater $\operatorname{ECV}(p=0.034)$, but similar native T1 values $(p=0.791)$.
Table 3 summarizes the results for the comparison of CMR and PET measurements using multivariate linear regression model by adjusting for confounding factors. The differences in CMR measurements of native T1, post T1 and ECV between subjects with moderate to heavy alcohol consumption and healthy controls remained statistically significant after adjusting for age, body mass index (BMI), history of smoking, and diastolic blood pressure (all $p<0.05$ ). In contrast, no significant differences were found in all CMR measurements

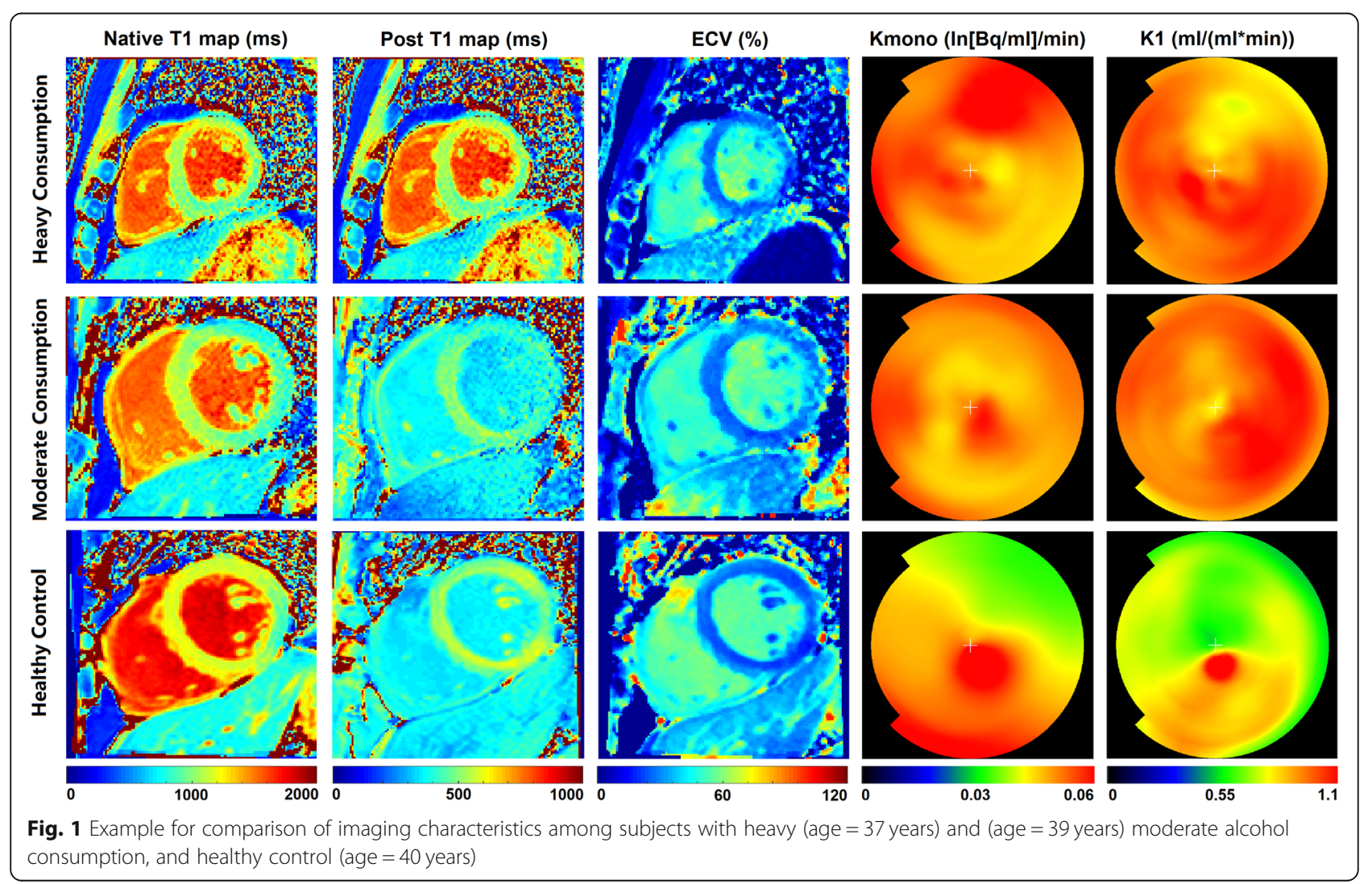


Table 3 Association between cardiac imaging measurements and alcohol consumption

\begin{tabular}{|c|c|c|c|c|c|c|}
\hline & \multicolumn{3}{|c|}{ Alcohol consumption vs. healthy controls ${ }^{a}$} & \multicolumn{3}{|c|}{ Heavy vs. moderate alcohol consumption ${ }^{\mathrm{b}}$} \\
\hline & $\beta^{c}$ & $95 \% \mathrm{Cl}$ & $P$ & $\beta^{c}$ & $95 \% \mathrm{Cl}$ & $P$ \\
\hline \multicolumn{7}{|l|}{ CMR measurements } \\
\hline Native T1, ms & -59.19 & $-89,-30$ & $<0.001$ & -8.83 & $-58,40$ & 0.714 \\
\hline Post T1, ms & -33.01 & $-57,-9$ & 0.008 & -21.16 & $-51,8$ & 0.155 \\
\hline $\mathrm{ECV}, \%$ & 1.63 & $0.58,2.67$ & 0.003 & 1.29 & $-0.25,2.83$ & 0.097 \\
\hline \multicolumn{7}{|l|}{ PET measurements } \\
\hline Kmono, $\min ^{-1} \times 10^{-3}$ & -10.67 & $-21.57,0.24$ & 0.055 & -10.93 & $-19.26,-2.61$ & 0.012 \\
\hline $\mathrm{K} 1, \min ^{-1}$ & -0.04 & $-0.17,0.09$ & 0.548 & -0.01 & $-0.12,0.09$ & 0.808 \\
\hline
\end{tabular}

ECV extracellular volume fraction. ${ }^{\mathrm{a}}$ The multivariate model is adjusted for age, BMI, history of smoke, and diastolic blood pressure. ${ }^{\mathrm{b}}$ The multivariate model is adjusted for age, and systolic blood pressure. 'Values are the difference in MRI and PET imaging measurements between subjects with moderate to heavy alcohol consumption and healthy controls and between subjects with heavy and moderate alcohol consumption

between subjects with heavy and moderate alcohol consumption after adjusting for age and systolic blood pressure (all $p>0.05$, Fig. 1).

\section{Comparison of PET measurements}

For the measurements from PET imaging, no significant differences in Kmono and K1 were observed between subjects with moderate to heavy alcohol consumption and healthy controls (all $p>0.05$, Table 2). Similarly, the Kmono of subjects with moderate alcohol consumption didn't significantly differ from those of healthy controls $(p=0.861)$. Meanwhile, subjects with heavy alcohol consumption showed significantly lower Kmono values compared with subjects with moderate alcohol consumption and healthy controls (all $p<0.05$, Fig. 1). No differences in $\mathrm{K} 1$ were found among subjects with moderate and heavy alcohol consumption and healthy controls (all $p>0.05$ ).

Table 4 Association between CMR and PET measurements

\begin{tabular}{|c|c|c|c|c|}
\hline \multirow{3}{*}{$\begin{array}{l}\text { MR } \\
\text { measurements }\end{array}$} & \multicolumn{4}{|c|}{ PET measurements } \\
\hline & \multicolumn{2}{|l|}{ Kmono } & \multicolumn{2}{|l|}{ K1 } \\
\hline & $r$ & $P$ & $r$ & $P$ \\
\hline \multicolumn{5}{|l|}{ Healthy controls } \\
\hline Native T1 & 0.248 & 0.437 & 0.394 & 0.205 \\
\hline Post T1 & -0.176 & 0.585 & 0.316 & 0.316 \\
\hline ECV & 0.343 & 0.274 & 0.689 & 0.013 \\
\hline \multicolumn{5}{|c|}{ Moderate alcohol consumption } \\
\hline Native T1 & -0.011 & 0.970 & 0.057 & 0.840 \\
\hline Post T1 & 0.045 & 0.874 & 0.211 & 0.451 \\
\hline ECV & 0.056 & 0.842 & 0.518 & 0.048 \\
\hline \multicolumn{5}{|c|}{ Heavy alcohol consumption } \\
\hline Native $\mathrm{T} 1$ & 0.440 & 0.059 & -0.263 & 0.276 \\
\hline Post T1 & 0.368 & 0.121 & 0.264 & 0.275 \\
\hline ECV & -0.207 & 0.395 & -0.072 & 0.768 \\
\hline
\end{tabular}

ECV extracellular volume
Multivariate linear regression analysis revealed that the differences in Kmono and K1 between subjects with alcohol consumption and healthy controls were not statistically significant after adjusting for confounding factors (all $p>0.05)$. In contrast, significant differences were found in Kmono $(p=0.012)$, but not in K1 $(p=0.808)$, between subjects with heavy and moderate alcohol consumption after adjusted for confounding factors (Table 3).

\section{Correlation between CMR and PET measurements}

The correlations between CMR and PET measurements among different groups are shown in Table 4. Strong and moderate correlations were found between $\mathrm{K} 1$ and ECV in healthy controls $(r=0.689, p=0.013)$ and subjects with moderate alcohol consumption $(\mathrm{r}=0.518, p=$ 0.048), respectively. No significant correlations were found between $\mathrm{K} 1$, native, and post T1 values, or between Kmono, native, and post $\mathrm{T} 1$ values (all $\mathrm{p}>0.05$ ).

\section{Reproducibility of PET measurements}

The inter-observer ICCs of Kmono and K1 were 0.997 (95\%CI: 0.993-0.999) and 0.977 (95\%CI: 0.944-0.991), respectively. The intra-observer ICCs of Kmono and K1 were 0.997 (95\%CI: $0.987-0.999)$ and 0.977 (95\%CI: $0.942-0.991)$, respectively.

\section{Discussion}

This study investigated the characteristics of tissues and metabolism in myocardium of asymptomatic subjects with moderate to heavy alcohol consumption using quantitative CMR and PET/CT imaging. We found that compared to healthy controls, subjects with moderate to heavy alcohol consumption showed a significant decline in native $\mathrm{T} 1$ and post $\mathrm{T} 1$ values, and an increase in ECV. Subjects with heavy alcohol consumption showed similar native T1, post T1, and ECV values measured by CMR but had significantly lower Kmono measured by PET, compared to subjects with moderate alcohol consumption after adjusting for confounding factors. A 
linear correlation was found between ECV and $\mathrm{K} 1$ in healthy controls but this correlation was weakened as daily alcohol consumption increased.

In the present study, subjects with moderate to heavy alcohol consumption showed significantly shorter native and post $\mathrm{T} 1$ values compared with healthy controls. The decline of native T1 of myocardium may reflect myocardial fat deposition in individuals with alcohol consumption. da Silva et al. found that alcoholism was significantly associated with fat deposition in the LV myocardium (OR: 0.161; 95\% CI: 0.072 to $0.36 ; p<0.05)$ [16]. Experimental studies have demonstrated that chronic alcoholism stimulates the oxidative/nitrative stress, impairs myocardial mitochondrial function and fatty acid metabolism, and leads to steatosis and fat deposition in the myocardium [17].

The lack of significant differences in native and post T1 values between subjects with heavy and moderate alcohol consumption may be explained by the evidence that cardiac steatosis is an early phenomenon which is largely amplified by binges and prone to attenuation by increased duration of alcohol consumption [17]. In addition, focal replacement fibrosis of the myocardium in patients with alcoholic cardiomyopathy may attenuate the shortened T1 values by fat deposition [18]. Animal studies have shown that alcohol exposure modulates cardiac fibroblast matrix metalloproteinases (MMPs) and tissue inhibitors of MMPs (TIMPs) expression and stimulates TGF- $\beta$ release from fibroblasts, favoring a profile associated with cardiac collagen accumulation $[19,20]$. Though our study showed that there was a difference in native $\mathrm{T} 1$ between subjects with moderate to heavy alcohol consumption in comparison analysis, this difference was attenuated after adjusting for confounding factors. This phenomenon indicates that this difference may be influenced by age, BMI, and history of smoking and diastolic blood pressure. Our findings suggest that $\mathrm{T} 1$ measurements may not be a sensitive indicator for impairment of the myocardium in subjects with different doses of alcohol consumption.

We found that ECV of subjects with moderate to heavy alcohol consumption was significantly greater than that of healthy controls, but the differences between subjects with heavy and moderate alcohol consumption were not significant after adjusting for confounding factors. An increased ECV usually reflects a non-specific expansion of extracellular space or indicates myocardial fibrosis or an abnormal deposition of metabolites into the myocardium. Previous evidence indicates that chronic alcohol exposure gives cells a greater tendency to undergoing apoptosis via increasing the reactive oxygen species (ROS), which are crucial mediators in signal transduction during cell apoptosis process $[21,22]$. The sequela of apoptosis, such as shrinkage and death of cardiomyocytes, may contribute to the increases in ECV in subjects with alcohol consumption. The mechanism for lack of differences in ECV between subjects with heavy and moderate alcohol consumption is unclear. As mentioned above, however, many pathophysiological changes are associated with the measurement of ECV.

In our study population, subjects with moderate alcohol consumption showed similar Kmono values to healthy controls, but this measurement was significantly reduced in those with heavy alcohol consumption. It has been shown that alcohol can inhibit mitochondrial respiration and the activity of enzymes in the tricarboxylic acid cycle, and interfere with both mitochondrial calcium uptake and binding synchronously [23]. The reduction of Kmono may therefore represent the inhibition of myocardial energy metabolism due to chronic alcohol consumption. Our subjects with moderate alcohol consumption having similar Kmono values to healthy controls may indicate compensatory sustaining of the myocardial metabolism, although there is apoptosis of cardiomyocytes caused by the toxicity of ethanol. Our findings suggest that Kmono measured by $11 \mathrm{C}$-acetate PET/CT imaging might be an effective marker for the changes of myocardial metabolism in asymptomatic subjects with moderate to heavy alcohol consumption.

In the healthy controls and subjects with moderate alcohol consumption, our data showed that there was a significant correlation between ECV and K1. It is expected that $\mathrm{K} 1$ is positively correlated with ECV in the healthy controls. Although there was expansion of ECV in subjects with heavy alcohol consumption, K1 that represent myocardial perfusion were unchanged.

In the current study, some subjects with moderate to heavy alcohol consumption may be at the asymptomatic stage of alcoholic cardiomyopathy. Though their cardiac function is normal, these subjects may be at higher risk of progressing to symptomatic stages if abstinence or dose control are not applied. Compared with CMR imaging, 11C-acetate PET/CT imaging can detect metabolic disorders of myocardium in subjects with a long history of alcohol consumption. As such, multimodal imaging may be an effective tool for stratifying the risk of cardiomyopathy caused by chronic alcohol consumption.

Our study has several limitations. First, this study only recruited asymptomatic subjects with moderate to heavy alcohol consumption. It will be valuable to determine the imaging features of symptomatic alcoholic cardiomyopathy patients. In addition, fat deposition within the myocardium might contribute to the decline of native $\mathrm{T} 1$ in subjects with alcohol consumption. However, fat quantification was not conducted in this study. Investigators utilized CMR techniques, such as fat-water separation imaging and H-CMR spectroscopy, 
to quantify cardiac fat deposition, and found that myocardial steatosis was a common phenomenon in idiopathic dilated cardiomyopathy and closely associated with myocardial triglyceride accumulation and diastolic dysfunction [24, 25]. Finally, only $1 / 3$ of healthy subjects completed the PET imaging due to exposure to ionizing radiation.

\section{Conclusion}

Asymptomatic men with moderate to heavy alcohol consumption have detectable changes in myocardial tissues and metabolism on quantitative CMR and $11 \mathrm{C}$-acetate $\mathrm{PET} / \mathrm{CT}$ imaging. Compared with quantitative CMR, $11 \mathrm{C}$-acetate PET/CT imaging may be more sensitive for detecting differences in myocardial damage among subjects with moderate to heavy alcohol consumption.

\begin{abstract}
Abbreviations
BMI: Body mass index; CMR: Cardiovascular magnetic resonance; CO: Cardiac output; ECM: Extracellular matrix; ECV: Extracellular volume; LV: Left ventricle/ left ventricular; LVEDV: Left ventricular end diastolic volume; LVEF: Left ventricular ejection fraction; LVESV: Left ventricular end systolic volume; LVSV: Left ventricular stroke volume; MBF: Myocardial blood flow; MMPs: Matrix metalloproteinases; $\mathrm{MVO}_{2}$ : Myocardial oxygen consumption; ROS: Reactive oxygen species; PET: Positron emission tomography; RVEDV: Right ventricular end diastolic volume; RVEF: Right ventricular ejection fraction; RVESV: Right ventricular end systolic volume; RVSV: Right ventricular stroke volume; TIMPs: Tissue inhibitors of MMPs
\end{abstract}

\section{Acknowledgments}

We greatly thank Mr. Zach Miller from Department of Radiology at the University of Washington, Seattle, WA, USA, for his help in language editing.

\section{Authors' contributions}

S.L., X.L., L.H., L.F. and X.Z. conceived and designed the study. X.L. and L.F. involved in the recruitment of the participants, the collection of the clinical data and screening with echocardiography. S.L., R.G. and H.D. acquired the MR data. S.L., X.W., F.S. and H.D. involved in the MR data analysis. X.S., J.D. and $H . X$ acquired the PET data. X.S., C.H., J.K. and L.H. involved in the PET data analysis. S.L. H. D and R. Z performed statistical analysis. S.L. and X. Z drafted the manuscript and L. H, X.L. and J.K. revised the manuscript. The author(s) read and approved the final manuscript.

\section{Funding}

This study was supported by grants from Philips Healthcare and funded by the grants of National Natural Science Foundation of China (81670349) and those of Beijing Natural Science Foundation (7172166).

\section{Availability of data and materials}

The CMR and 11C-acetate PET/CT data used and/or analyzed in the present study are available from the corresponding author on reasonable requests.

\section{Ethics approval and consent to participate}

The study protocol was approved by Institutional Review Board of Tsinghua University School of Medicine and the written consent form was obtained from all subjects prior to enrollment.

\section{Consent for publication}

All authors have read and approved the final version of this manuscript.

\section{Competing interests}

The authors declare that they have no competing interests.

\section{Author details}

'Center for Biomedical Imaging Research, Department of Biomedical Engineering, Tsinghua University School of Medicine, Haidian District, Beijing
100084, China. ${ }^{2}$ Department of Cardiology, Peking Union Medical College Hospital, Chinese Academy of Medical Science, Beijing, China. ${ }^{3}$ Department of Nuclear Medicine, Peking Union Medical College Hospital, Chinese Academy of Medical Science, 1\# Shuaifuyuan, Dongcheng District, Beijing 100730, China. ${ }^{4}$ Beijing Key Laboratory of Molecular Targeted Diagnosis and Therapy in Nuclear Medicine, Beijing, China. ${ }^{5}$ Department of Biomedical Engineering, Beijing Institute of Technology School of Life Science, Beijing, China. ${ }^{6}$ Turku PET Center, Turku University Hospital and University of Turku, Turku, Finland. ${ }^{7}$ Department of Neurology, Beijing Tiantan Hospital, Capital Medical University, Beijing, China. ${ }^{8}$ Department of Medical Engineering, First Affiliated Hospital of PLA General Hospital, Beijing, China.

Received: 6 September 2019 Accepted: 3 March 2020

Published online: 16 April 2020

\section{References}

1. Mokdad AH, Marks JS, Stroup DF, Gerberding JL. Actual causes of death in the United States, 2000. JAMA. 2004;291(10):1238-45.

2. Rehm J, Mathers C, Popova S, Thavorncharoensap M, Teerawattananon Y, Patra J. Global burden of disease and injury and economic cost attributable to alcohol use and alcohol-use disorders. Lancet. 2009;373(9682):2223-33.

3. Shield KD, Rylett M, Gmel G, Gmel G, Kehoe-Chan TA, Rehm J. Global alcohol exposure estimates by country, territory and region for 2005 -- a contribution to the comparative risk assessment for the 2010 global burden of disease study. Addiction. 2013;108(5):912-22.

4. Fauchier L, Babuty D, Poret P, Casset-Senon D, Autret ML, Cosnay P, et al. Comparison of long-term outcome of alcoholic and idiopathic dilated cardiomyopathy. Eur Heart J. 2000;21(4):306-14.

5. Skotzko CE, Vrinceanu A, Krueger L, Freudenberger R. Alcohol use and congestive heart failure: incidence, importance, and approaches to improved history taking. Heart Fail Rev. 2009;14(1):51-5.

6. Abramson JL, Williams SA, Krumholz HM, Vaccarino V. Moderate alcohol consumption and risk of heart failure among older persons. JAMA. 2001; 285(15):1971-7.

7. Djoussé L, Gaziano JM. Alcohol consumption and risk of heart failure in the Physicians' health study I. Circulation. 2006;115:34-9.

8. Piano MR, Phillips SA. Alcoholic cardiomyopathy: pathophysiologic insights. Cardiovasc Toxicol. 2014;14:291-308.

9. Cunningham CC, Spach PI. Alcoholism and myocardial energy metabolism. Alcohol Clin Exp Res. 1994;18(1):132-7.

10. Moon JC, Messroghli DR, Kellman P, Piechnik SK, Robson MD, Ugander M, et al. Myocardial T1 mapping and extracellular volume quantification: a Society for Cardiovascular Magnetic Resonance (SCMR) and CMR working Group of the European Society of cardiology consensus statement. J Cardiovasc Magn Reson. 2013;15:92

11. Braunschweig F, Sörensen J, von Bibra H, Olsson A, Rydén L, Långström B, et al. Effects of biventricular pacing on myocardial blood flow and oxygen consumption using carbon-11 acetate positron emission tomography in patients with heart failure. Am J Cardiol. 2003;92(1):95-9.

12. Yoshinaga K, Ohira $H$, Tsujino I, Oyama-Manabe N, Mielniczuk L, Beanlands $\mathrm{RS}$, et al. Attenuated right ventricular energetics evaluated using ${ }^{11} \mathrm{C}$-acetate PET in patients with pulmonary hypertension. Eur J Nucl Med Mol Imaging. 2014;41:1240-50.

13. Kitaizumi $K$, Yukiiri $K$, Masugata $H$, Takinami $H$, Iwado $Y$, Noma $T$, et al. Acute improvement of cardiac efficiency measured by 11C-acetate PET after cardiac resynchronization therapy and clinical outcome. Int J Cardiovasc Imag. 2010;26:285-92.

14. Sun KT, Yeatman LA, Buxton DB, Chen K, Johnson JA, Huang SC, et al. Simultaneous measurement of myocardial oxygen consumption and blood flow using [1-carbon-11] acetate. J Nucl Med. 1998;39(2):272-80.

15. Cerqueira MD, Weissman NJ, Dilsizian V, Jacobs AK, Kaul S, Laskey WK et al. Standardized myocardial segmentation and nomenclature for tomographic imaging of the heart: a statement for healthcare professionals from the cardiac imaging Committee of the Council on clinical cardiology of the American Heart Association. Circulation. 2002; 105(4):539-42.

16. da Silva RMS, de Mello RJV. Fat deposition in the left ventricle: descriptive and observacional study in autopsy. Lipids Health and Dis. 2017;16:86.

17. Matyas C, Varga ZV, Mukhopadhyay P, Paloczi J, Lajtos T, Erdelyi K, et al. Chronic plus binge ethanol feeding induces myocardial oxidative stress, 
mitochondrial and cardiovascular dysfunction, and steatosis. Am J Physiol Heart Circ Physiol. 2016:310(11):H1658-70.

18. Hookana E, Junttila MJ, Puurunen VP, Tikkanen JT, Kaikkonen KS, Kortelainen $\mathrm{ML}$, et al. Causes of nonischemic sudden cardiac death in the current era. Heart Rhythm. 2011;8(10):1570-5.

19. El Hajj EC, El Hajj MC, Voloshenyuk TG, Mouton AJ, Khoutorova E, Molina PE, et al. Alcohol modulation of cardiac matrix metalloproteinases (MMPs) and tissue inhibitors of MMPs favors collagen accumulation. Alcohol Clin Exp Res. 2014;38(2):448-56.

20. Law BA, Carver WE. Activation of cardiac fibroblasts by ethanol is blocked by TGF- $\beta$ inhibition. Alcohol Clin Exp Res. 2013:37(8):1286-94.

21. Hajnóczky G, Buzas CJ, Pacher P, Hoek JB, Rubin E. Alcohol and mitochondria in cardiac apoptosis: mechanisms and visualization. Alcohol Clin Exp Res. 2005;29(5):693-701.

22. Guan Z, Lui CY, Morkin E, Bahl JJ. Oxidative stress and apoptosis in cardiomyocyte induced by high-dose alcohol. J Cardiovasc Pharmacol. 2004; 44(6):696-702.

23. Bing RJ. Cardiac metabolism: its contributions to alcoholic heart disease and myocardial failure. Circulation. 1978;58(6):965-70.

24. Lu M, Zhao S, Jiang S, Yin G, Wang C, Zhang Y, et al. Fat deposition in dilated cardiomyopathy assessed by CMR. JACC Cardiovasc Imaging. 2013; 6(8):889-98.

25. Utz W, Engeli S, Haufe S, Kast P, Hermsdorf M, Wiesner S, et al. Myocardial steatosis, cardiac remodelling and fitness in insulin-sensitive and insulinresistant obese women. Heart. 2011;97(19):1585-9.

\section{Publisher's Note}

Springer Nature remains neutral with regard to jurisdictional claims in published maps and institutional affiliations.

Ready to submit your research? Choose BMC and benefit from:

- fast, convenient online submission

- thorough peer review by experienced researchers in your field

- rapid publication on acceptance

- support for research data, including large and complex data types

- gold Open Access which fosters wider collaboration and increased citations

- maximum visibility for your research: over $100 \mathrm{M}$ website views per year

At $\mathrm{BMC}$, research is always in progress.

Learn more biomedcentral.com/submissions 